ORIGINAL ARTICLE

\title{
Infection with Ureaplasma urealyticum: is there a specific clinical and radiological course in the preterm infant?
}

\author{
U Theilen, A J Lyon, T Fitzgerald, G M A Hendry, J W Keeling
}

Arch Dis Child Fetal Neonatal Ed 2004;89:F163-F167. doi: 10.1136/adc.2003.026013

See end of article for authors' affiliations

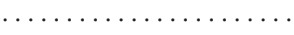

Correspondence to: Mr Lyon, Simpson Centre for Reproductive Health, Royal Infirmary of Edinburgh, Little France, Edinburgh EH16 4SA, Scotland, UK; andrew.lyon@ luht.scot.nhs.uk

Accepted 15 April 2003

\begin{abstract}
Background: Despite having mild early respiratory disease, many preterm babies develop chronic lung disease (CLD). Intrauterine infection with Ureaplasma urealyticum has been associated with preterm labour and CLD.

Objective: To test the hypothesis that infection with $U$ urealyticum results in a specific clinical and radiological picture in the first 10 days of life.

Methods: Retrospective study of 60 ventilated babies $<30$ weeks gestation, who had tracheal secretions tested for $U$ urealyticum. Placental histology was reviewed by a paediatric pathologist for signs of chorioamnionitis. Chest radiographs were independently reviewed by two paediatric radiologists according to previously agreed criteria. All reviewers were blinded to the infection status of the babies. Results: Twenty five babies were $U$ urealyticum positive. These were more likely to experience chorioamnionitis $(p=0.004)$, premature rupture of membranes $(p=0.01)$, and spontaneous vaginal delivery $(p=0.09)$. U urealyticum positive babies had fewer signs of respiratory distress syndrome on early chest radiographs ( $p=0.038$ ), and they could be weaned from their ventilation settings (fraction of inspired oxygen $\left(\mathrm{FIO}_{2}\right)$ and mean airway pressure) more quickly in the first few days. Subsequently $U$ urealyticum positive babies deteriorated clinically and radiologically. More often they required ventilation to be restarted $(p=0.051)$, a higher proportion being ventilated on day $10(p=0.027)$ with higher $\mathrm{FIO}_{2}$ ( $p=0.001)$ and mean airway pressure $(p=0.002)$. Their chest radiographs showed more emphysematous changes as early as day $5(p=0.045)$, with a pronounced difference by day 10 ( $p=0.009$ ).

Conclusions: Preterm ventilated babies with $U$ urealyticum in their tracheal secretions have a different clinical and radiological course, with less acute lung disease but early onset of CLD, compared with those with negative cultures.
\end{abstract}

p he severity of early lung disease in the preterm infant has diminished. This has been due, in part, to improved perinatal and neonatal care, in particular antenatal steroids, replacement surfactant, and better ventilatory techniques. Despite the improvement in early lung disease, many very immature babies go on to develop chronic lung disease (CLD), with persistent oxygen dependency. ${ }^{1}$ The character of CLD has changed since the classical description of bronchopulmonary dysplasia by Northway. At that time, relatively mature infants were affected after exposure to high pressure ventilation and high levels of inspired oxygen. ${ }^{2}$ CLD is now seen in extremely low birthweight babies after relatively minor early lung disease.

Intrauterine infection is a recognised factor in the development of CLD. ${ }^{3}$ Chorioamnionitis has been associated with less acute lung disease but a higher incidence of chronic lung problems. ${ }^{4}$ The genital mycoplasmas, in particular Ureaplasma urealyticum, are the most commonly isolated organisms in the genital tract of mothers. ${ }^{5} U$ urealyticum has been shown to cause chronic inflammation and early fibrosis in the preterm lung, ${ }^{6}$ and has been associated with the development of CLD. ${ }^{78}$

Our policy has been to culture all ventilated infants below 30 weeks gestation for the genital mycoplasmas. If the organism is isolated, the babies are treated with erythromycin for 14 days.

It was our impression that babies with $U$ urealyticum in their tracheal aspirates had a specific clinical and radiological course, compared with those who remained culture negative. They appeared to have less acute respiratory disease but an earlier progression to CLD associated with specific radiological features, particularly cystic changes.

The aim of this study was to test the hypothesis that infection with $U$ urealyticum at birth resulted in a specific clinical and radiological picture in the first 10 days of life of the preterm baby.

Ethical approval was obtained from the Lothian research ethics committee.

\section{METHODS}

Babies delivered in the Royal Infirmary of Edinburgh under 30 weeks gestation, between January 1999 and end of December 2000, who were ventilated for at least 24 hours and had tracheal secretions sent for culture of $U$ urealyticum, were included. Those with major congenital abnormality were excluded.

Tracheobronchial secretions were obtained, during the first week, after the instillation of $0.5 \mathrm{ml}$ saline down the endotracheal tube. The secretions were transported to the laboratory immediately and cultured for all common pathogens. For $U$ urealyticum, a specific broth was used (PPLO broth; Difco Laboratories, Detroit, Michigan, USA) enriched with $1 \%$ urea. This organism possesses a urease that breaks down urea to ammonia and raises the $\mathrm{pH}$ of the broth, which is detected using a phenol red indicator. Each sample was incubated at a temperature of $37^{\circ} \mathrm{C}$ for up to five days. Positive samples were recultured on blood agar to detect possible contamination by other urea splitting organisms. This method had previously been validated using polymerase chain reaction. ${ }^{9}$ 
Clinical and ventilation data from the first 10 days of life were obtained by reviewing medical and nursing notes.

Placental histology was reviewed for features of chorioamnionitis. The pathologist was blind to the infection status and clinical course of the baby.

All babies had routine chest radiography but this was performed at different times depending on clinical need. Radiographs were divided into three time groups: day 1, those taken on day 1 or 2; day 5, taken on days 3-6; day 10 , taken on days 7-14. Random radiographs from these time periods were reviewed independently by two paediatric radiologists, both blind to the infection status and the clinical course of the baby. Radiographs were scored for diffuse granular changes indicative of acute respiratory distress syndrome, alveolar changes characteristic of emphysema or cysts, and interstitial changes associated with developing fibrosis. The film was divided into four quadrants, and each feature scored as 0 (absent) or 1 (present) in each quadrant. This gave a total score of $0-4$ for each feature in each radiograph.

$\chi^{2}$ and Wilcoxon rank tests were used to compare the groups.

The level of agreement between the two radiologists was assessed using Cronbach's $\alpha$ test of reliability.

Logistic regression was used to explore the association between the development of significant emphysematous change on the radiographs on day 10 and gestation, birth weight, $U$ urealyticum, prolonged rupture of membranes, and chorioamnionitis.

\section{RESULTS}

In the two year period, there were 89 eligible inborn babies. Secretions had been cultured for $U$ urealyticum in 60 . Twenty five were $U$ urealyticum positive, and 35 were negative. There were no differences between the groups with respect to proportion of boys or multiple deliveries. In each group, $75 \%$ of the mothers had received at least one dose of antenatal steroids, and all babies were given replacement surfactant. In 54 babies, the surfactant was given within 15 minutes of delivery. There was no difference between the groups with respect to total number of doses of surfactant.

The babies in the $U$ urealyticum positive group had a significantly lower gestational age. There was a trend to a lower birth weight but this did not reach significance (table 1).

Preterm premature rupture of membranes (more than 24 hours before delivery) was significantly more common in the $U$ urealyticum positive group. There was also a trend towards vaginal delivery being more common in the $U$ urealyticum positive group (table 1 ).

Placental histology was available in 24 of the $U$ urealyticum positive and 30 of the negative group. Table 1 shows that

Table 1 Patients and perinatal course

\begin{tabular}{|c|c|c|c|}
\hline & $\begin{array}{l}\text { U urealyticum } \\
\text { positive }\end{array}$ & $\begin{array}{l}U \text { urealyticum } \\
\text { negative }\end{array}$ & p Value \\
\hline Number & 25 & 35 & \\
\hline Gestation (weeks) & $25(25-26)$ & $26(25-27.5)$ & $0.016^{*}$ \\
\hline Birth weight $(\mathrm{g})$ & $770(710-900)$ & $890(700-985)$ & $0.085^{\star}$ \\
\hline PPROM & $13(52)$ & $7(21)$ & $0.01 \dagger$ \\
\hline Chorioamnionitis & $13(54)$ & $5(17)$ & $0.004 \uparrow$ \\
\hline $\begin{array}{l}\text { Spontaneous vaginal } \\
\text { delivery }\end{array}$ & $17(68)$ & $16(46)$ & $0.09+$ \\
\hline \multicolumn{4}{|c|}{$\begin{array}{l}\text { Values are median (interquartile range) or number (\%). } \\
\text { PPROM, Preterm premature rupture of membranes. } \\
\text { *Wilcoxon test. } \\
+\chi^{2} \text { test. }\end{array}$} \\
\hline
\end{tabular}

Table 2 Early clinical course

\begin{tabular}{|c|c|c|c|}
\hline & $\begin{array}{l}U \text { urealyticum } \\
\text { positive }\end{array}$ & $\begin{array}{l}U \text { urealyticum } \\
\text { negative }\end{array}$ & $\mathrm{p}$ Value \\
\hline Maximum WCC & $28(24-44)$ & $19(14-29)$ & 0.015 \\
\hline $\begin{array}{l}\text { Maximum neutrophil } \\
\text { count }\end{array}$ & $20(16-34)$ & $13(8-20)$ & 0.013 \\
\hline Day of maximum WCC & $8(2-9)$ & $9(4-10)$ & 0.087 \\
\hline Day of lowest $\mathrm{FlO}_{2}$ & $2(2-3)$ & $3(2-6)$ & 0.052 \\
\hline Day of lowest MAP & $4(3-6)$ & $6(4-9)$ & 0.039 \\
\hline
\end{tabular}

Values are median (interquartile range). $\mathrm{p}$ Value was obtained with the Wilcoxon test.

WCC, White cell count; $\mathrm{FIO}_{2}$, fraction of inspired oxygen; MAP, mean

airway pressure.

chorioamnionitis was significantly more common in the $U$ urealyticum positive group. Of the 18 mothers in the study who had evidence of chorioamnionitis, $13(72 \%)$ of the babies grew $U$ urealyticum in the tracheal secretions.

Table 2 shows that the $U$ urealyticum positive group had significantly higher total white cell and neutrophil counts during the first 10 days. There was a trend for an earlier peak of the white cell count in $U$ urealyticum positive babies. This did not reach significance. There was no difference between the groups in the occurrence of positive cultures in tracheal secretions or blood for organisms other than $U$ urealyticum. Other than erythromycin, which was given to all culture positive babies, there was no difference in antibiotic use in the two groups.

All babies were weaned from ventilation over the first few days. In both groups, the minimum fraction of inspired oxygen $\left(\mathrm{FIO}_{2}\right)$ was $22-23 \%$, and the mean airway pressure was $5 \mathrm{~cm} \mathrm{H}_{2} \mathrm{O}$. These minimum values were achieved significantly earlier in the $U$ urealyticum group, despite these babies being less mature (table 2 ).

By 10 days of age, significantly more of the $U$ urealyticum positive group needed to be reventilated, after a period of extubation of at least 24 hours (table 3 ). By this age, more of the positive group were still on a ventilator and they needed significantly higher levels of inspired oxygen and mean airway pressures than those who were $U$ urealyticum negative (table 3).

The difference between the two groups on day 10 could have been caused by the relative immaturity of the $U$ urealyticum positive babies. Looking at the subgroup of 39 babies under 27 weeks gestation, there was no difference in the gestational age between the $U$ urealyticum positive $(\mathrm{n}=21)$ and the $U$ urealyticum negative $(\mathrm{n}=18)$ babies. Still, the $U$ urealyticum positive babies in this subgroup needed more support on day 10. There was a significant difference in the $\mathrm{FIO}_{2}$ (median 42 (interquartile range 31-48) $v 27(23-34), \mathrm{p}=0.011)$. In the subgroup, more

Table 3 Clinical course after 10 days

\begin{tabular}{llll}
\hline & $\begin{array}{l}\text { U urealyticum } \\
\text { positive }\end{array}$ & $\begin{array}{l}\text { U urealyticum } \\
\text { negative }\end{array}$ & p Value \\
\hline $\begin{array}{l}\text { Reventilated after } \\
24 \text { hours off }\end{array}$ & $8(32)$ & $4(11)$ & $0.051^{*}$ \\
$\begin{array}{l}\text { ventilator } \\
\text { Still ventilated on }\end{array}$ & $19(75)$ & $16(55)$ & $0.027^{*}$ \\
day 10 & & & \\
$\mathrm{FIO}_{2}$ on day 10 & $39(31-47)$ & $25(23-32)$ & $0.001 \dagger$ \\
$\mathrm{MAP}$ on day 10 & $7(6-8)$ & $5(4-7)$ & $0.002 \dagger$ \\
\hline
\end{tabular}

Values are number (\%) or median (interquartile range).

${ }^{*} \chi^{2}$ test.

tWilcoxon test.

$\mathrm{FlO}_{2}$, Fraction of inspired oxygen; MAP, mean airway pressure. 
$U$ urealyticum positive babies were still ventilated (16 $v 9$, $\mathrm{p}=0.089)$ and needed a higher mean airway pressure (median 7 (interquartile range 6-8) v 5 (3-8), p = 0.076).

Looking at the outcome beyond 10 days of life in the entire study group, the total number of days of ventilation was significantly higher in the $U$ urealyticum group (median 33 days (interquartile range 10-39) $v 12$ days (5-22), $\mathrm{p}=0.015)$. More patients from the positive group were given postnatal steroids $(7 \vee 2, \mathrm{p}=0.015)$.

At 36 weeks corrected gestation, there was no significant difference between the groups $(91 \%$ in the survivors who were culture positive compared with $82 \%$ in those who were culture negative).

There were no differences between the groups with respect to need for inotropes or incidence of patent ductus arteriosus, necrotising enterocolitis, or intraventricular haemorrhage. In the $U$ urealyticum positive group, there was a trend towards an increase in periventricular leucomalacia, but this did not reach significance $(4 v \mathrm{l}, \mathrm{p}=0.072)$.

\section{Radiology}

The level of agreement between the radiologists was good for all features, with Cronbach's $\alpha$ of 0.84 for diffuse granularity, 0.9 for emphysematous changes, and 0.9 for interstitial changes. For further analysis, the scores from the two radiologists were averaged.

Table 4 shows the results for the three time periods. The $U$ urealyticum negative group showed significantly more changes characteristic of respiratory distress syndrome, as shown by the degree of diffuse granularity, on the first radiographs (fig 1). By day 5 the $U$ urealyticum positive group was showing early emphysematous changes (fig 2), which were more obvious by day 10 and significantly different from the $U$ urealyticum negative babies (fig 3 ).

There was an increasing trend to interstitial changes in fibrosis in the $U$ urealyticum positive group by day 10, but this did not reach significance.

\section{Logistic regression}

$U$ urealyticum colonised infants were less mature and had a higher incidence of chorioamnionitis and prolonged rupture of membranes. In a logistic regression model using emphysematous change on day 10 as the dependent variable with gestation, birth weight, $U$ urealyticum, preterm prolonged rupture of membranes, and chorioamnionitis as covariates, only $U$ urealyticum and chorioamnionitis remained as independent variables.

Table 4 Radiograph scores for the three time periods

\begin{tabular}{|c|c|c|c|}
\hline & $\begin{array}{l}\text { U urealyticum } \\
\text { positive }\end{array}$ & $\begin{array}{l}\text { U urealyticum } \\
\text { negative }\end{array}$ & p Value \\
\hline \multicolumn{4}{|l|}{ Day 1} \\
\hline No of radiographs & 22 & 30 & \\
\hline Diffuse granularity & $1(0-3)$ & $3(0-4)$ & 0.038 \\
\hline Emphysema & $0(0-0)$ & $0(0-0)$ & 0.88 \\
\hline Interstitial changes & $0(0-0)$ & $0(0-0)$ & 0.89 \\
\hline \multicolumn{4}{|l|}{ Day 5} \\
\hline No of radiographs & 20 & 27 & \\
\hline Diffuse granularity & $2(0-3)$ & $1(0-3)$ & 0.35 \\
\hline Emphysema & 1 (0-2) & $0(0-1)$ & 0.045 \\
\hline Interstitial changes & $0(0-0)$ & $0(0-0)$ & 0.58 \\
\hline \multicolumn{4}{|l|}{ Day 10} \\
\hline No of radiographs & 20 & 24 & \\
\hline Diffuse granularity & $0(0-2)$ & $0(0-2)$ & 0.75 \\
\hline Emphysema & $1(0-3)$ & $0(0-1)$ & 0.009 \\
\hline Interstitial changes & $0(0-4)$ & $0(0-0)$ & 0.14 \\
\hline
\end{tabular}

Values are median (interquartile range). $\mathrm{p}$ value obtained using the Wilcoxon test.
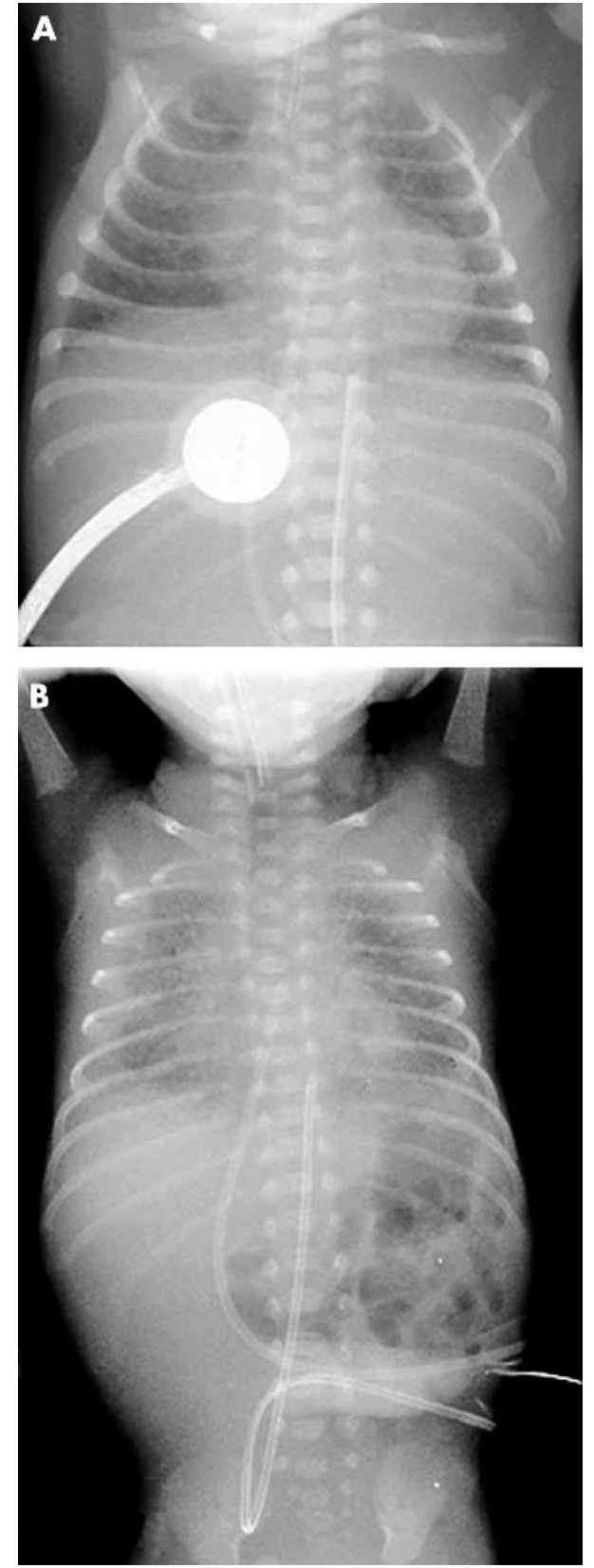

Figure 1 Chest radiograph of $(\mathrm{A})$ a Ureaplasma urealyticum positive baby and (B) a $U$ urealyticum negative baby on day 1 .

\section{DISCUSSION}

This was a retrospective study and not all ventilated babies were cultured for $U$ urealyticum. It is possible that those not cultured included a number of $U$ urealyticum positive babies who were well and had no significant radiological changes. However, in those cultured, the presence of $U$ urealyticum in tracheal secretions resulted in a significantly different clinical and radiological course from similar babies who were culture negative.

The mothers of the $U$ urealyticum positive babies had a higher incidence of preterm rupture of membranes, were more likely to have chorioamnionitis on placental histology, and vaginal delivery was more common. There was an $80 \%$ chance of finding $U$ urealyticum in tracheal secretions if there was histological evidence of chorioamnionitis and preterm premature rupture of membranes or spontaneous vaginal delivery. This increased to $90 \%$ for babies of 24-26 weeks 

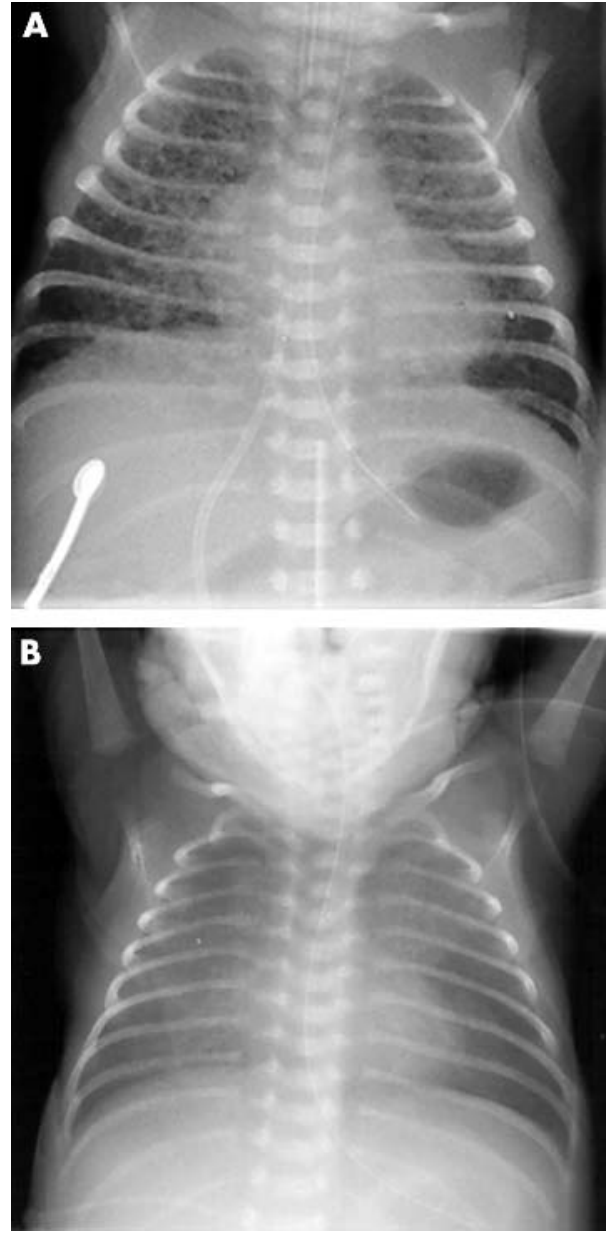

Figure 2 Chest radiograph of (A) a Ureaplasma urealyticum positive baby and (B) a $U$ urealyticum negative baby on day 5 .

gestation. A higher proportion of $U$ urealyticum infection in less mature preterm babies has been shown in other studies. ${ }^{10}$

Despite the significantly lower gestation of the $U$ urealyticum positive group, these babies had milder acute lung disease, with less widespread radiographic changes associated with respiratory distress syndrome and more rapid weaning of initial ventilation. By 10 days, however, the positive group had worse respiratory problems than the negative group. This was confirmed in the subgroup of babies of less than 27 weeks gestation, where no difference in the gestational age was observed. This suggests that the clinical difference is an effect of infection with $U$ urealyticum. Clinical deterioration correlated with radiological changes associated with emphysema seen in the $U$ urealyticum positive group.

Others have reported an association of chorioamnionitis with less severe acute but more chronic lung disease. ${ }^{4}$ Antenatal infection matures the fetal lung at the expense of a disturbance in long term alveolar development. This is exacerbated after delivery by persistent inflammation in the airways, which is the precursor of chronic lung damage. ${ }^{11}$ It is not surprising that $U$ urealyticum and chorioamnionitis remain covariates in a logistic regression model, as $U$ urealyticum is a common cause of ascending infection in the mother and is often a factor in the preterm onset of labour. In this group of babies, the development of significant emphysematous radiographic change by day 10 was independent of gestation.

There is no universally accepted scoring system for neonatal radiographs, although other workers have used
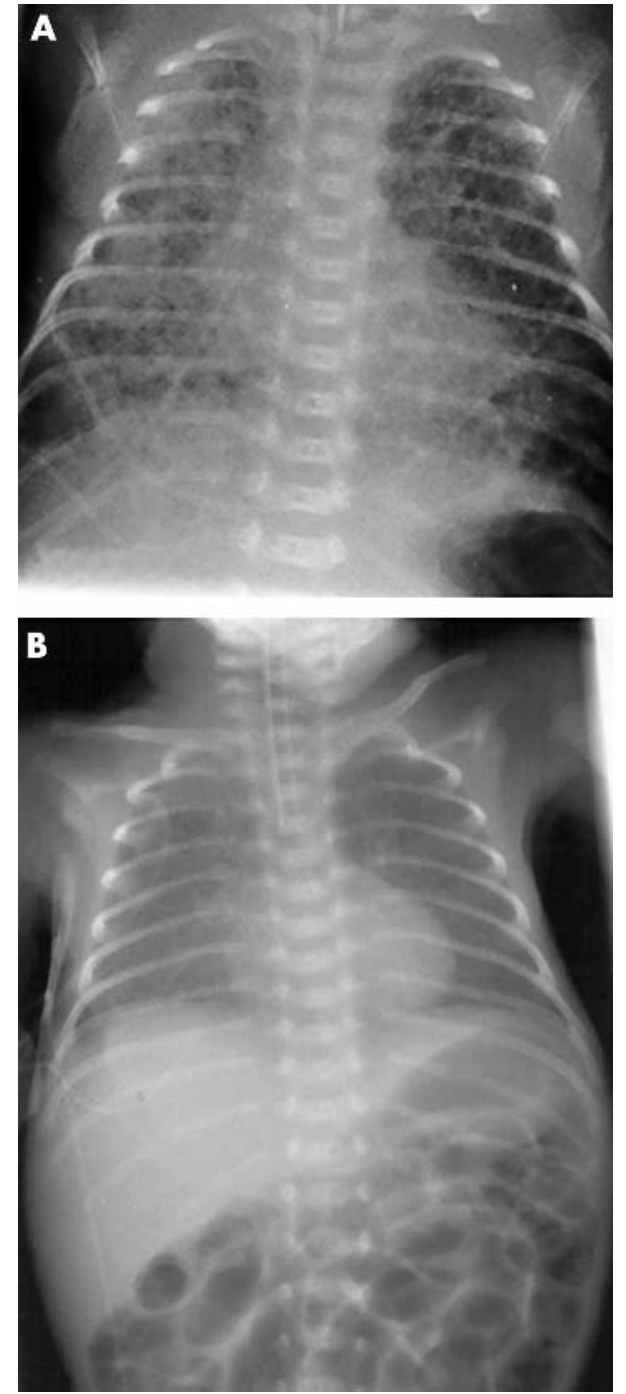

Figure 3 Chest radiograph of $(\mathrm{A})$ a Ureaplasma urealyticum positive baby and (B) a $U$ urealyticum negative baby on day 10 .

similar systems to the one devised for this study. ${ }^{12}$ There was very good agreement between the two independent radiologists for all the features, and we believe that the scoring system gave an accurate representation of the radiographic changes in these babies. The obvious changes in the $U$ urealyticum positive group suggest quite significant lung damage in these babies within the first 10 days. Crouse et $a l^{13}$ have previously reported a higher incidence of pneumonia and precocious bronchopulmonary dysplasia in radiographs of neonates under $1250 \mathrm{~g}$ birth weight who had U urealyticum in tracheal aspirates.

Despite the clinical and radiological deterioration seen by day 10 , we did not find a significant increase in the number of babies in the culture positive group who developed chronic lung disease, as defined by oxygen requirement at 36 weeks corrected gestation. However, this was a small, retrospective study and not powered to look at the incidence of chronic lung disease.

$U$ urealyticum is an important causative factor in preterm labour and development of chronic lung damage in the immature infant. The ORACLE study ${ }^{14}$ showed a slight benefit for the baby if mothers received erythromycin, but only if there was premature preterm rupture of membranes. There was no reduction in chronic lung disease alone, defined as oxygen dependence at 36 weeks gestation, but there was 
improvement in the composite outcome, which included CLD as one of the components.

At present, there is no good evidence that treatment of the baby after delivery reduces the risk of chronic lung damage. ${ }^{15}$ It may be that antenatal infection has already activated the inflammatory cascade and started the lung injury sequence. Treatment after delivery may be too late. However, with a deteriorating clinical and radiological picture, it is difficult not to try to eradicate any organisms found in the respiratory tract, at least until better evidence is available from clinical trials.

\section{Authors' affiliations}

U Theilen, A J Lyon, T Fitzgerald, Simpson Centre for Reproductive Health, Royal Infirmary, Edinburgh, Scotland, UK

G M A Hendry, J W Keeling, Royal Hospital for Sick Children, Edinburgh

\section{REFERENCES}

1 Rojas A, Gonzalez, Abancalari E, et al. Changing trends in the epidemiology and pathogenesis of neonatal chronic lung disease. $J$ Pediatr 1995; 126:605-10.

2 Northway WH, Rosan RC, Porter DY. Pulmonary disease following respiratory therapy of hyaline membrane disease: bronchopulmonary dysplasia. N Engl J Med 1967;276:357-68.

3 Lyon A. Chronic lung disease of prematurity. The role of intra-uterine infection. Eur J Pediatr 2000;159:798-802.
4 Watterberg KL, Demers LM, Scott SM, et al. Chorioamnionitis and early lung inflammation in infants in whom bronchopulmonary dysplasia develops. Pediatrics 1996;97:210-15.

5 Cassell GH, Waites KB, Watson H-L, et al. Ureaplasma urealyticum intrauterine infections: role in prematurity and disease in newborns. Clin Microbiol Rev 1993;6:69-87.

6 Viscardi RM, Manimtim WM, Sun C-C J, et al. Lung pathology in premature infants with Ureaplasma urealyticum infection. Pediatr Dev Pathol 2002; 5: 141-50.

7 Cassell GH, Waites KB, Crouse DT. Perinatal mycoplasmal infections. Clin Perinatol 1991; 18:241-62.

8 lles R, Lyon A, Ross P, et al. Infection with Ureaplasma urealyticum and Mycoplasma hominis and the development of chronic lung disease in preterm infants. Acta Paediatr 1996;85:482-4.

9 Cunliffe NA, Fergusson S, Davidson F, et al. Comparison of culture with polymerase chain reaction for detection of Ureaplasma urealyticum in endotracheal aspirates of preterm infants. Clin Microbiol 1996;45:27-30.

10 Romero R, Mazor K, Wu Y, et al. Infection in the pathogenesis of preterm labour. Semin Perinatol 1988:12:262-79.

11 Jobe $\mathbf{A H}$, Ikegami M. Mechanisms initiating lung injury in the preterm. Early Hum Dev 1998;53:81-94.

12 Greenough A, Thomas $M$, Johnson A, et al. Prediction of chronic lung disease from the chest radiograph appearance at seven days of age. Early Hum Dev 2003;73:931-1109.

13 Crouse DT, Odrezin GT, Cutter GR, et al. Radiological changes associated with tracheal isolation of Ureaplasma urealyticum from neonates. Clin Infect Dis 1993;17(suppl 1):S122-30.

14 Kenyon SL, Taylor DJ, Tarnow-Mordi W, et al. Broad-spectrum antibiotics for preterm, prelabour rupture of fetal membranes: the ORACLE 1 randomised trial. Lancet 2001;357:979-88.

15 Lyon AJ, McColm J, Middlemist L, et al. Randomised trial of erythromycin on the development of chronic lung disease in preterm infants. Arch Dis Child Fetal Neonatal Ed 1998;78:F10-14. 